\title{
Comparision of observed and theoretical amplitudes of oscillations above granules and intergranular lanes
}

\author{
E. V. Khomenko \\ Main Astronomical Observatory, National Academy of Sciences, \\ Golosiiv, Kyiv-127, Ukraine, 03680
}

\begin{abstract}
We do modeling of the wave propagation in the solar photosphere. NLTE synthesis of the time series of the Fe I $5324 \AA$ line profiles is performed using 3D model atmosphere. Velocity and intensity oscillations resulted from computations are compared with high spatial resolution observations. We conclulde that differences in oscillatory amplitudes above granules and intergranular lanes can be produced by variations of the physical conditions in these structures without invoking any excitation mechanisms.
\end{abstract}

\section{Model of oscillations}

The idea of this paper is to investigate how variations of physical conditions in solar photosphere affect the propagating wave.

We solved analytically the hydrodynamical equations of mass, momentum and energy conservation. We assumed that there are no magnetic field; viscosity and thermal conduction terms can be omitted; the effects of solar rotation are negligible; to the $0^{\text {th }}$ order the atmosphere is plane parallel, horizontally homogeneous and in radiative equilibrium. The matter is described by equation of state neglecting ionization effects. The effects of radiative relaxation are taken into account by Newtonian cooling approximation. The motion is restricted to one vertical dimension. The atmosphere was aproximated as a series of 102 isothermal layers with constant convetive velocity. In each layer we linearized the equations and let the disturbance propagate in a form of a plane wave:

$$
V_{1}, T_{1}, \rho_{1} \sim e^{z\left(1 \pm k_{i}\right)} e^{ \pm i k_{r}\left( \pm t V_{p h}-t V_{0}-z\right)}
$$

where $k_{r}$ and $k_{i}$ are imaginary and real parts of the vertical wave number, $V_{p h}$ is the phase velocity of the wave and $V_{0}$ is the convective velocity. Here we use only upward solution by selecting the upper signes in the Eq. 1 . We computed velocity, temperature and density perturbations for all isothermal layes. These perturbations were introduced into the model atmosphere and profiles of the Fe I $5324 \AA$ line were synthesized. In the present study we use a snapshot of the 3D model from the most recent numerical simulations by Asplund et al. (2000). The simulated box is $6.0 \times 6.0 \times 3.8 \mathrm{Mm}$ large with nearly $1.0 \mathrm{Mm}$ being located above the continuum optical depth unity. For the line profiles calculations we chosed one slice from the snapshot and extracted a set of $501 \mathrm{D}$ granular and intergranular models. Line profiles were synthesised using NLTE 

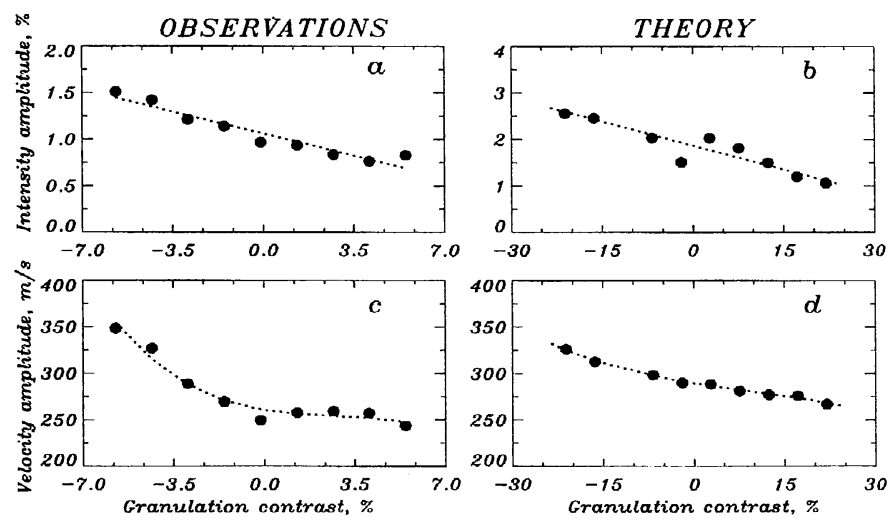

Figure 1. Amplitudes of the line center intensity $(a$ and $b)$ and velocity ( $c$ and $d$ ) oscillations as a function of granulation contrast. Left column: observed amplitudes; right column: calculated amplitudes. Positive values of contrast mean granules and negative are intergranular lanes.

departure coefficients found for each model from the self-consistent solution of the kinetic and radiative transfer equations (Shchukina \& Trujillo Bueno, 2000). The overall computational time was one wave period. Initial amplitude of the wave perturbation was chosen equal $100 \mathrm{~m} \mathrm{~s}^{-1}$ at height $0 \mathrm{~km}$, with the period beeing $270 \mathrm{~s}$. For each of the 50 time series we calculated line center velocity and intensity oscillations and measured their amplitudes. The granulation contrast of a $j^{\text {th }}$ series was defined as $\left(I_{c j}-\bar{I}_{c}\right) / \bar{I}_{c}$, where $\bar{I}_{c}$ is the averaged continuum intensity over all 50 series.

\section{Observations}

We compared the results of our computations with high spatial resolution observations obtained with German VTT at the Observatorio del Teide, Tenerife. The CCD images contain the photospheric line Fe I $5324 \AA$. The observed area $\left(\sim 0.38 \times 89 \operatorname{arcsec}^{2}\right)$ was placed near the quiet solar disk centre. The spectrograms were taken every $9.3 \mathrm{~s}$ during 31 minutes. The spatial resolution of this observations was about 0.5 arcsec. For every spatial pixel row we inferred line center velocity and intensity and continuum intensity variations. This variations are caused mainly by convective and wave motions. We separated them using $k-\omega$ diagram. We restricted the wave motions by temporal frequency range $\omega=1.8-5.7 \mathrm{mHz}$ and spatial frequency lower $0.18 \mathrm{Mm}^{-1}(\lambda>5500 \mathrm{~km})$. Convective motions were limited only in the temporal frecuency $\omega<2.2 \mathrm{mHz}$. Here we study the relationships between oscillatory component of the line center velocity and intensity and the convective component of continuum intensity variations. 


\section{Results}

The results of our computations in comparision with observations are displayed in Fig. 1. Observed amplitudes were measured as extreme values in each oscillating element. Granulation contrast was also determined at those moments of time. After that we averaged amplitudes for the certain contrast intervals. The averaged amplitudes show a clear dependence on granulation contrast. Intensity amplitude grows linearly nearly two times as the contrast changes from $5.5 \%$ to $-5.5 \%$. The largest amplitudes are observed above intergranular lanes and they are larger above the darkest lanes. Velocity amplitude depends slightly on the granulation contrast and increases only 1.4 times over the same interval. So, at average, the large-amplitude oscillations are suppressed above granules.

Theoretical velocity and intensity amplitudes are displayed in panels $b$ and $d$ of the Fig. 1. As we see, theory also gives an increase of the amplitudes above intergranular lanes. Teoretical intensity amplitudes are about 2 times larger above the lanes than above granules and velocity amplitudes are 1.3 times larger. This is very similar to observations. The main difference between left and right panels of Fig. 1 is that the contrast range is larger and the amplitudes are higher in theory. We believe that this is caused by better spatial resolution of the 3D model (125 km in comparision with no more than $500 \mathrm{~km}$ in observations). High resolution observations show larger values of granulation contrast. Outstanding stratospheric observations of solar granulation by Karpinsky (1990) with resolution of $0^{\prime \prime} 2(\approx 200 \mathrm{~km})$ reveal the value of contrast as large as $22 \%$. Thus, we can compare results in the Fig. 1 assuming that observed and calculated ranges of contrast correspond each other.

\section{Conclusions}

We conclude that even with all initial conditions beeing equal at the bottom of the atmosphere, oscillations above granules and intergranular lanes turn out to have different amplitudes at heights about $500 \mathrm{~km}$, where the core of our Fe I line is formed. This changes are produced by modulation of the propagating wave by atmospheric imhomogeneties. The more extensive analysis performed by us in Khomenko et al. (2000) supports this finding.

Acknowlegements. I am grateful to R. Kostik for his help with observations analysis and useful discussions. Special thanks to M. Asplund for the kind permission to use 3D model atmosphere for the purposes of this study.

\section{References}

Asplund, M., Nordlund, A., Trampedach, R., Prieto, C. A., Stein, R. F. 2000, A\&A, 359, 729

Karpinsky, V. N. 1990 in IAU Symp. 138, Structure, Convection and Magnetic Fields, ed. J.-O. Stenflo (Dordrecht: Kluwer), 23

Khomenko, E. V., Kostik, R. I., Shchukina, N. G. 2000, A\&A, submitted

Shchukina, N. G., Trujillo Bueno, J. 2000, ApJ, submitted 